\title{
Blast Injuries: Biophysics, Pathophysiology and Management Principles
}

\author{
CL Horrocks
}

\begin{abstract}
"The blast wave is a shot without a bullet, a slash without a sword. It is present everywhere within its range. Blast would be as dreaded a weapon as chemical warfare, if its range, when explosives are used were not limited to small areas. However it would be premature to believe that this situation will always remain the same."
\end{abstract}

Theodor Benzinger 1950

\section{Introduction}

Explosions are physical, chemical or nuclear reactions that involve the rapid release of considerable amounts of energy. Their deleterious effects on living organisms are embodied by the term blast injury.

The incidence of injuries resulting from explosions has increased throughout the twentieth century. This has been caused in part by industrial expansion. More significant has been the proliferation of explosive weapons and their increased use world-wide. Injuries caused by explosions are not confined to the battlefield. The International Committee of the Red Cross has estimated that throughout 70 countries, at least 2,000 people (predominantly civilians) are killed or injured every month by anti-personnel mines. From 1990 to 1995, 15,700 criminal bombing incidents were recorded in the United States by the Federal Bureau of Investigation (FBI). These resulted in the deaths of 355 persons and injuries to 3176. Explosions caused by industrial accidents and terrorist devices are infrequent, but unpredictable events in developed countries. Therefore, civilian doctors may be required to deal with large numbers of blast injured subjects with little warning in chaotic and difficult circumstances. An understanding of the mechanisms of these injuries and their management is useful to all physicians. Explosions and the injuries caused by them are the subjects of this paper.

Lt Col C L Horrocks FRCS (ORL-HNS)

RAMC

Dept of

Otorhinolaryngology

Southampton

University Hospitals

NHS Trust,

Southampton General

Hospital,

Tremona Road,

Southampton,

Hants, SO16 6DY

\section{Explosions}

Terrorist devices and military ordnance typically contain high explosives. A high explosive (e.g. trinitrotoluene, gelignite, Semtex) is a compound (solid or fluid) which can undergo an intense exothermic reaction, releasing large amounts of energy very quickly. This process propagates at supersonic speeds (approximately $5000 \mathrm{~m} / \mathrm{s}^{-}$) radially from the explosive and is termed detonation. A shock wave (blast wave) is generated in the surrounding air and resulting variations in air pressure set in motion the mass movement of air (the dynamic overpressure or blast wind). The shock wave, the dynamic overpressure and thermal energy are the underlying phenomena which contribute to blast injuries.

By convention, blast injuries are classified according to the mechanism by which they are produced (Table 1).

Table 1. Classification of Blast Injuries.

\begin{tabular}{ll}
\hline Type of Blast Injury & Mechanism \\
\hline PRIMARY & Interaction of the blast wave \\
& with the body. Gas containing \\
& structures (ear, lungs, gastro- \\
& intestinal tract) are particularly \\
& at risk. \\
& Bomb fragments and other \\
projectiles energised by the & explosion cause penetrating and \\
& non-penetrating wounds. Any \\
& part of the body may be \\
& affected. \\
& Displacement of the body (or of \\
& its constituent parts). This \\
& mechanism contributes to the \\
& traumatic amputation of limbs. \\
& Structural collapse of buildings, \\
& e.g. crush injuries. \\
& A miscellaneous collection of all \\
other mechanisms such as: & Flash burns (superficial burns \\
& to exposed skin caused by the \\
& radiant and convective heat of \\
& the explosion). \\
& Methaemoglobinaemia due to \\
poisoning by dinitrobenzene or & potassium perchlorate \\
(components of WWI & munitions) (Laure 1993). \\
Acute septicaemic melioidosis \\
due to inhalation of soil \\
particles contaminated with \\
Pseudomonas pseudomallei \\
(Wang et al 1993). \\
Explosions are associated with a \\
high incidence of psychological \\
sequelae in injured and \\
uninjured survivors. \\
\end{tabular}

A detailed discussion of the generation and propagation of blast waves and their interaction with structures is given elsewhere in this volume (Cullis). An overview is presented below.

The variation in blast wave pressure with time, at a fixed point in space (the Friedlander relationship) describes the 
physical properties of the blast wave (Iremonger 1997). At the time of arrival of the shock-front, the pressure increases effectively instantaneously to a maximum (peak overpressure), from which it falls exponentially to sub-atmospheric levels and returns to the ambient pressure (Figure 1).

Table 2. Explosive Devices.

\begin{tabular}{ll}
\hline Type & Pattern of Injury \\
\hline CONVENTIONAL WEAPONS & All types of blast injury may occur, but \\
e.g. grenades, aerial bombs, mortar & penetrating injuries from multiple \\
bombs, rockets. & fragments predominate. Primary \\
& fragments are derived from the \\
& munition, preformed within the shell or \\
& from the casing when the munition \\
& explodes. Other materials (building \\
& debris, vehicle components) energised \\
& by the blast form secondary fragments.
\end{tabular}

\section{TERRORIST DEVICES}

Vary from a few to several hundred kilograms of explosive.
Although the reported incidence of primary blast injuries varies from $1 \%$ (Hill 1979) - 76\% (Katz et al 1989), serious primary blast injury is uncommon and secondary and tertiary injuries predominate. Mortality is low (up to $5 \%$ ) unless the device is large, explodes in a confined space or there is structural collapse. Less than $50 \%$ of those presenting to hospital will require admission.

\section{ANTIPERSONNEL MINES}

Common in developing countries. Indiscriminate in action. Tend to maim adults, but may kill (particularly children).

Patterns of injury include:

Traumatic amputation of foot or leg due to standing on a buried "point is required to detonate some of these detonating" mine. As little as $5 \mathrm{~kg}$ weight devices. Mine fragments, grass, soil, parts of shoe and foot are blown upwards with substantial proximal tissue damage and contamination.

A more random distribution of penetrating injuries caused by fragment mines triggered near victim by a tripwire. Severe upper limb and facial and ocular injuries due to handling of a mine.

\section{ENHANCED-BLAST MUNITIONS}

e.g. fuel-air explosives (a distributed energy source such as a vapour). effect rather than by framentation. Used by Soviet forces in Afghanistan.

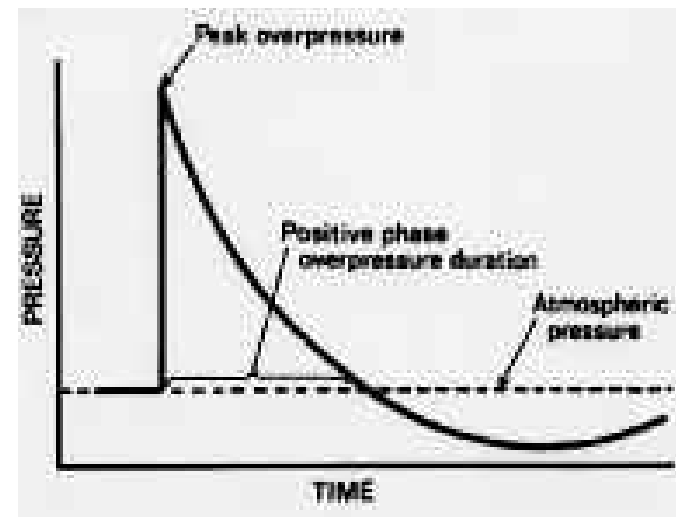

Fig 1. Idealised blast overpressure waveform.

The biological effects of the blast wave depend on the peak overpressure and the positive phase duration. This pressure time relationship represents an ideal free field blast wave in air. In practice, environmental features such as buildings and walls within enclosed spaces reflect the incident wave generating complex overpressures.

In air, the intensity of the blast wave decreases rapidly as the blast wave expands and its radius of effect is smaller than that of fragments set in motion by the blast. Therefore primary blast injuries in the open tend to be confined to the relative proximity of the epicentre and are less common than ballistic (secondary) injuries. A casualty close enough to an explosion to sustain serious primary injuries will commonly have lethal secondary and tertiary injuries. The majority of survivors of an explosion will have sustained secondary or tertiary injuries, the management of which follows conventional principles. Military ordnance and terrorist devices are specifically designed to injure by generating fragments on detonation and therefore maximise casualties (Table 2 and Table 3). These secondary injuries must be treated systematically.

Primary blast injuries particularly affect
Table 3. Terrorist Devices.

\begin{tabular}{ll}
\hline Type & Pattern of Injury \\
\hline CAR BOMBS & $\begin{array}{l}\text { Traumatic amputation of lower limbs } \\
\text { Typically } 1-3 \mathrm{~kg} \text { of commercial }\end{array}$ \\
$\begin{array}{l}\text { asplosive positioned under the floor of } \\
\text { and often containing fragments of metal }\end{array}$ \\
the car directly beneath the driver's seat. & $\begin{array}{l}\text { and vehicle upholstery. } \\
\text { and }\end{array}$
\end{tabular}

\section{BARE CHARGES}

e.g. Booby traps.

Small improvised devices $(<10 \mathrm{~kg})$

detonated remotely by wire or radio signal.

\section{LORRY OR VAN BOMBS}

Large devices $(>40 \mathrm{~kg})$. Detonation

results in formation of large secondary

missiles (from the body of the vehicle).

Extensive penetrating injuries and gross disruption of the body.

\section{CULVERT BOMBS}

Very large devices designed to disrupt passing vehicles. Vehicle may be displaced and victims ejected.
Primary blast injuries and ballistic injuries due to secondary fragments are characteristic.

Crush injuries from secondary damage to buildings.

Gross disruption and disintegration of the body. gas-containing structures. Solid organs including the skin are more resistant to the blast wave. Therefore in pure primary blast injury, a patient may display little external evidence of trauma. This situation is best illustrated in cases of immersion blast where water propagates the shock wave effectively, but inhibits the movement of projectiles.

Underwater explosions generate a large volume of energetic gaseous products (underwater bubble) and a shockwave which is propagated into the surrounding water. This is reflected as a negative pressure wave at the water surface, which is disrupted forming a dome of minute water droplets (spall). The reflected tension wave interacts with the compressive shock wave and accelerates its decay. An individual treading water at the time of an underwater explosion will therefore experience a higher impulse (the integral of pressure over time) on the lower portion of the body because the reflected tension wave (cut-off wave) arrives 
later. Regions of the body which are deeper in the water are more severely affected by the blast wave. It is however a misconception that abdominal injuries predominate in exposure to underwater blast irrespective of depth. Individuals at risk of immersion blast are therefore safer floating on the surface of the water rather than treading water in an upright position.

Under particular circumstances there may be a higher incidence of serious primary blast injury:

- In enclosed spaces (vehicles or buildings) where reflection of the blast wave augments the total blast load;

- Where the victim is close to the explosion;

- In individuals wearing body armour which confers protection against fragments, but not the blast wave;

- With large explosions;

- With fuel-air explosives and other types of enhanced blast munitions (Dearden in this volume).

Primary blast injuries are unfamiliar to most physicians; this review concentrates on this group of injuries.

\section{The Biophysics of Primary Blast Injury}

Primary blast injuries are caused by the interaction of the blast wave with the body and are therefore a type of non-penetrating trauma. Non-penetrating injuries from impact may be direct or indirect. Direct

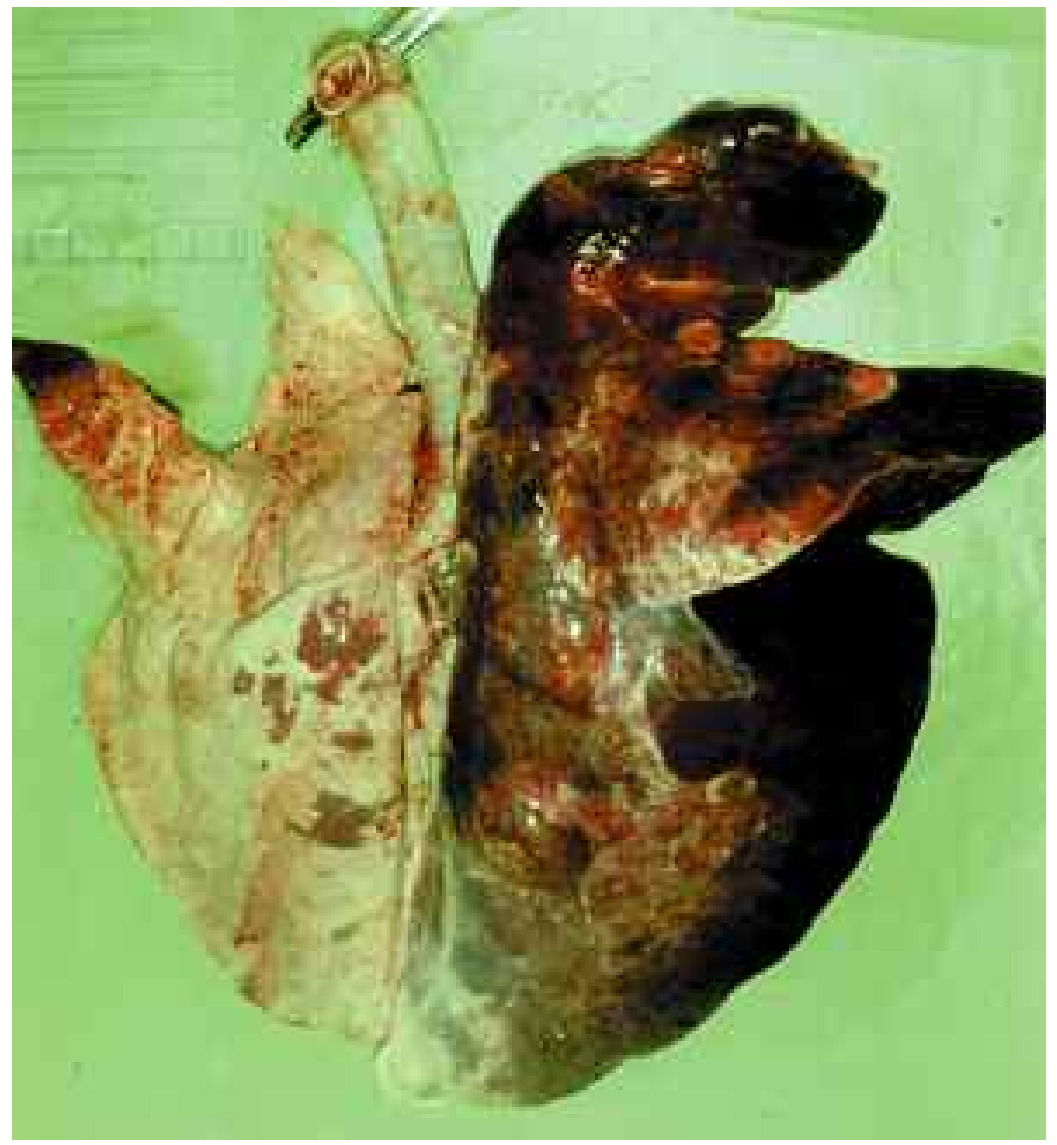

Fig 2. Primary blast injury to pig lung. injuries occur in close proximity to the region of body wall deformation and are generally due to local shear. Indirect injuries are caused by transmitted stress or shear waves and occur at locations spatially separated from the site of body wall displacement. Primary blast injuries belong to both groups - there may be contusion in parenchyma associated with the body wall, but also centrally .

Interaction of the blast wave with the body wall generates two types of waves (Cooper et al 1989):

\section{Stress Waves}

These are longitudinal pressure waves like sound. They travel at or slightly faster than the speed of sound, but differ from sound because of their high amplitude and velocity. Shock waves are a special form of high pressure stress wave, with an effectively instantaneous wavefront, which travel through a medium at speeds greater than the speed of sound in that medium. Stress waves have a number of properties which help explain their effects on tissues:

- They generate high local forces with small, but rapid distortions. Pathophysiological effects are at the microvascular level; gross lacerations are not typical.

- They affect organs with marked differences in physical properties (acoustic impedance) and therefore particularly involve those which contain gas.

- Reflection and reinforcement occurs at interfaces causing augmentation of pressures far from the site of body impact (stress concentration).

The coupling of stress waves through the thoracic and abdominal walls is responsible for blast lung and primary blast injury of the small bowel respectively. Stress waves probably injure tissues in a number of ways:

- Pressure differentials across delicate structures such as alveolar septa.

- When a stress wave encounters an interface between two media of different physical properties, for example when it attempts to propagate from bowel wall tissues into the gas filled lumen, a component of the compressive stress wave is reflected back at the interface as a tension wave. Most materials are weaker in tension than compression and disruption at the interface (tissue damage) may result.

- A stress wave may compress a gascontaining structure such as an alveolus or bowel segment (implosion); subsequent expansion damages the wall of the structure.

Shear Waves

These are long duration, low velocity, transverse waves, resulting from deformation of the body wall and compression of the visceral structures. Asynchronous movements of tissues of differing inertia 


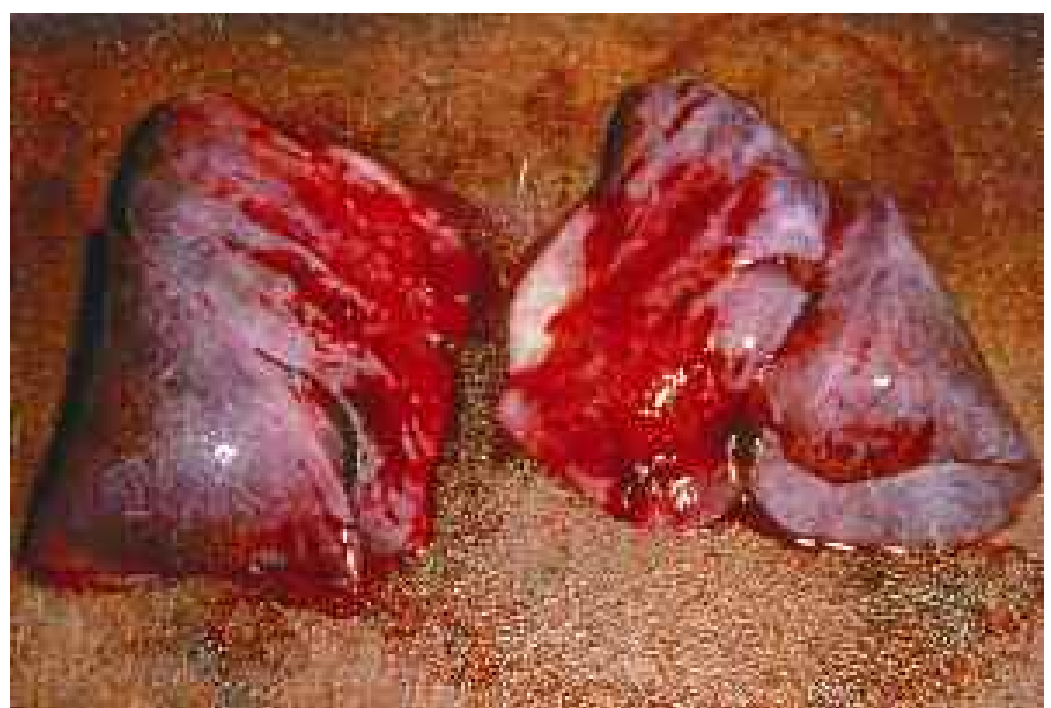

Fig 3. "Rib" markings in human lung injured by blast wave.

cause tearing of structures from their attachments and shearing of solid organs. Shear waves are probably responsible for primary blast injury of solid abdominal viscera, mesenteries and the large bowel.

\section{The Respiratory Tract \\ The Lung}

(Pulmonary Primary Blast Injury; Blast Lung) Early mortality and severe morbidity due to primary blast injury are determined by the severity of damage to the lungs.

\section{Pathology}

The blast wave disrupts inter-alveolar septa, the principal effect of which is haemorrhage into the alveoli and bronchioles. Depending on the blast load, this varies from scattered petechiae to large confluent haemorrhages involving the whole lung (Figure 2). Lacerations of the parenchyma are a feature in severely affected lungs. Certain regions of the lung are consistently more severely involved, for example lung in proximity to the mediastinum (particularly the azygos lobe) and in the costophrenic angles. There is also a predilection for the lateral lung surfaces in contact with the intercostal spaces giving rise to the erroneously described 'rib markings' (Figure 3). It has been proposed that concentration of stress waves due to reflection and augmentation within the thorax explains this unequal distribution.

When alveoli rupture near to the lung surface, subpleural cysts form. Tearing of the visceral pleura will result in a pneumothorax or haemopneumothorax (if the underlying lung is lacerated). Traumatic alveolo-venous fistulae permit the entry of air into pulmonary venules and the formation of systemic air emboli.

On light microscopy, pulmonary capillaries are seen to be dilated (for reasons that are not understood). There may be separation of alveolar tissue from bronchovascular elements with bleeding into the connective tissues which surround these, forming ring haemorrhages (Zuckerman 1940).

Experiments on rats subjected to blast revealed increased pinocytosis, blebbing and ballooning in pulmonary capillary endothelial and Type I epithelial cells on electron microscopy. Loss of structure or enlargement were occasionally noted in the lamellated bodies of Type II epithelial cells (Brown et al 1993). These ultrastructural features occurred not only in association with changes which were evident on light microscopy, but also in apparently normal regions of lung parenchyma. i.e. there may be patients in whom clinical and radiological evidence of blast lung is absent, at least initially, but who have sustained lung injury. The natural history of these lesions is unknown and it remains open to speculation whether they predispose to the development of adult respiratory distress syndrome (ARDS).

\section{Pathophysiology}

Pulmonary function in blast lung bears similarities to that in other types of nonpenetrating lung injury. Haemorrhage into alveoli and focal pulmonary oedema cause a ventilation perfusion mismatch (with increased intrapulmonary shunt), and reduced lung compliance, resulting in hypoxia and increased work of breathing (Cohn 1997).

Anecdotal reports from the nineteenth and early twentieth centuries have described individuals dying immediately following blast exposure and in whom little evidence of external injury was apparent. These observations have led to the concept that the blast wave causes an acute cardiovascular and respiratory response. Experimental work on animals subjected to thoracic blast has demonstrated a reflex triad of apnoea, bradycardia and hypotension (Guy et al 1998). It is thought that this triad is mediated by pulmonary afferent C-fibre receptors (formerly J-receptors), located in alveolar interstitial spaces close to pulmonary capillaries. An increase in pulmonary interstitial pressure or volume (due to pulmonary haemorrhage or oedema) could distort and therefore stimulate pulmonary Cfibres. Alternatively serotonin (released from platelets in blast damaged lung) might stimulate pulmonary afferent C-fibres. Pulmonary C-fibres are thought to be unmyelinated parasympathetic afferents transmitted in the vagus to the nucleus of the solitary tract. It is likely that this reflex operates in humans, but the degree to which it contributes to early morbidity and death has not been established.

\section{Clinical Features of Blast Lung}

The diagnosis of blast lung is made clinically and confirmed by chest X-ray. The clinical features are summarised in Table 4. Some case reports have suggested that blast lung may not manifest until $24-48$ hours after 
blast exposure (Coppel 1976). More commonly, patients with pulmonary primary blast injury have clinical and radiological evidence of blast lung on admission (Caseby et al 1976; Leibovici et al 1999).

Table 4. Clinical Features of Blast Lung.

\begin{tabular}{ll}
\hline Symptons & Dyspnoea \\
& Cough (dry to productive with frothy \\
& sputum) \\
& Haemoptysis \\
& Chest pain or discomfort (typically \\
& retrosternal) \\
\hline Signs & Tachypnoea \\
& Cyanosis \\
& Reduced breath sounds and dullness to \\
& percussion \\
& Coarse crepitations, rhonchi \\
& Features of pneumothorax or \\
& haemopneumothorax \\
& Subcutaneous emphysema \\
Retrosternal crunch (pneumomediastinum) & Retinal artery emboli
\end{tabular}

\section{MANAGEMENT}

\section{Initial Management}

Initial management follows conventional guidelines.

\section{Airway Maintainance With Cervical Spine Control}

The airway should be assessed to determine patency, cleared and maintained. The jaw thrust or chin lift manoeuvre may be sufficient in some patients. In the unconscious patient with an absent gag reflex an oropharyngeal airway can be used. If the patient is conscious an oropharyngeal airway is unlikely to be tolerated and a nasopharyngeal airway is more appropriate. In a patient with multiple injuries, it must be assumed that the cervical spine is injured, particularly with an altered level of consciousness or blunt injury above the clavicle. Appropriate measures must be taken to ensure immobility of the cervical spine.

\section{Breathing with Oxygenation}

The patient must be assessed for adequacy of ventilation and clinical evidence of blast lung. If the patient is apnoeic and at point of wounding immediately following injury, artificial ventilation is required by mouth or bag-valve-facemask apparatus. Artificial ventilation is not appropriate if there has been apnoea for more than a few minutes. Definitive control of the airway and ventilation is achieved by endotracheal intubation, but equipment and expertise may not be available. If intubation is not possible and the airway is obstructed an attempt should be made to establish a surgical airway. Patients with blast lung are at risk of pulmonary barotrauma and if there is evidence of tension pneumothorax, it should be decompressed immediately. All patients should receive oxygen at high flow rate (15 litres per minute). It should be noted that, on the battlefield, basic life support is not currently endorsed for treatment at point of wounding. In the majority of cases, apnoeic casualties will already be dead unless the potential rescuer was present at the time of wounding and was uninjured.

\section{Circulation With Control Of External Haemorrhage}

The cardiovascular status must be assessed. Significant external haemorrhage should be controlled by the application of direct pressure. Two widebore $(14-16 \mathrm{G})$ intravenous cannulae should be inserted and intravenous fluid administered judiciously. Patients with blast lung may be at risk from injudicious fluid replacement, which should therefore be closely monitored.

\section{Disability}

A rapid neurological assessment must be performed in order to determine the level of consciousness, pupillary size and reactions. Neurological abnormalities may be due to arterial air emboli. It can be difficult to assess the level of consciousness in a patient deafened by blast.

\section{Exposure}

Exposed patients with multiple injuries are at risk of developing hypothermia and care should be taken after removing the patient's clothing. A brief assessment should be made to exclude any injury which is immediately life threatening.

\section{Subsequent Management}

A chest radiograph is mandatory in all patients exposed to blast (Table 5).

Table 5. Radiological Evidence of Blast Lung

Diffuse pulmonary opacities "infiltrates". (Typically, these develop within a few hours, become maximal at $24-48$ hours and resolve over 7 days). Infiltrates which progress after 48 hours are likely to represent complications of blast lung, e.g. adult respiratory distress syndrome (ARDS) or pneumonia.

Pneumothorax or haemopneumothorax.

Interstitial (peribronchial) emphysema (long linear peribronchial lucency)

Subcutaneous emphysema.

Pneumomediastinum.

Pneumoperitoneum (usually secondary to perforation of an abdominal viscus, but tension

pneumoperitoneum has been reported in association with blast lung) (Oppenheim et al 1998).

Computed tomography (CT) of the thorax is not presently a routine investigation in the management of blast lung, but probably has a role in selected patients. Studies on nonblast pulmonary contusion have shown that CT has higher sensitivity than plain chest radiography in detecting early parenchymal and pleural lesions (Schild et al 1989), and a predictive role in determining which patients will require mechanical ventilation (Wagner 
et al 1989). The general management of blast lung is similar to that of pulmonary contusion. In particular blast lung casualties are susceptible to reduced lung function from injudicious fluid replacement. The high risk of pulmonary barotrauma (notably pneumothorax and systemic air emboli) in blast lung distinguishes it from other forms of pulmonary injury. Other manifestations of pulmonary barotrauma include interstitial emphysema, pneumomediastinum, subcutaneous emphysema, pneumoretroperitoneum and pneumoperitoneum.

It has been argued that positive pressure ventilation (PPV), particularly with high level positive end-expiratory pressure (PEEP) increases the risk of air embolism and pneumothorax and should therefore be avoided in blast casualties. Patients are similarly at risk during general anaesthesia and evacuation by air in partially pressurised aircraft. However, there will be patients for whom the severity of lung injury renders PPV mandatory. Various strategies have been proposed to reduce the likelihood or effects of pulmonary barotrauma. These have included:

- Prophylactic insertion of chest drains;

- Reversion to spontaneous breathing as soon as possible using intermittent mechanical ventilation (IMV) and continuous positive airway pressure (CPAP) (Uretzky et al 1980);

- Reduction of tidal volume and therefore peak inspiratory pressure with permissive hypercapnia (Sorkine et al 1998).

Less conventional techniques (independent lung ventilation, high frequency jet ventilation, nitric oxide inhalation and extracorporeal membrane oxygenation) have been employed in limited numbers of severely affected patients with varying degrees of success (Pizov et al 1999). Extracorporeal membrane oxygenation is a highly sophisticated technique and requires anticoagulation; it is probably incompatible with severe haemorrhagic lung injury. Independent lung ventilation has been used in patients with unilateral bronchopleural fistulae.

The prognosis for patients with uncomplicated blast lung seems to be favourable. In a small series of 11 patients with blast lung of whom 10 required mechanical ventilation, clinical pulmonary function tests were normal at one year (Hirshberg et al 1999).

\section{Non-Parenchymal Injury}

Rib fractures may occur in the posterior angles with high blast loads, but are uncommon and suggest secondary or tertiary injury. The mucosa of the upper and lower airways is sensitive to blast. When blast exposure is repetitive, damage occurs at overpressures below those responsible for pulmonary primary blast injury. Changes may occur throughout the airways, from the nasal fossae and paranasal sinuses to the bronchioles. These include loss of cilia, flattening of epithelial cells, epithelial stripping and mucosal haemorrhages.

Within the larynx, the vestibule, posterior epiglottic surface and arytenoids are particularly affected by mucosal bruising. Stripping of the epithelium off the basal lamina (the stripped-epithelium lesion) is the most common airway injury, resulting in ulceration and bleeding into the lumen. It is postulated that spalling is responsible for fragmentation of the epithelium at the tissueair interface (Chiffelle 1966). These injuries and intraluminal haemorrhage are potential causes of airway obstruction.

Cases of difficult intubation or acute airway compromise due to structural changes or bleeding have not been reported. In theory, mucosal injuries should render mucociliary clearance less effective. Whether they predispose patients with blast lung to secondary infection is not known. Mucosal lesions of the airways generally resolve spontaneously. They are sentinel injuries, indicating possible blast injury of the lung or at other sites and should be sought on examination of the upper respiratory tract.

\section{The Circulatory System}

\section{The Heart}

Direct injuries to the heart include haematomas and lacerations. Epicardial haematomas tend to be located on the diaphragmatic surface and endocardial contusions around the papillary muscles. Involvement of the myocardium is less frequent. Anteroseptal myocardial infarction

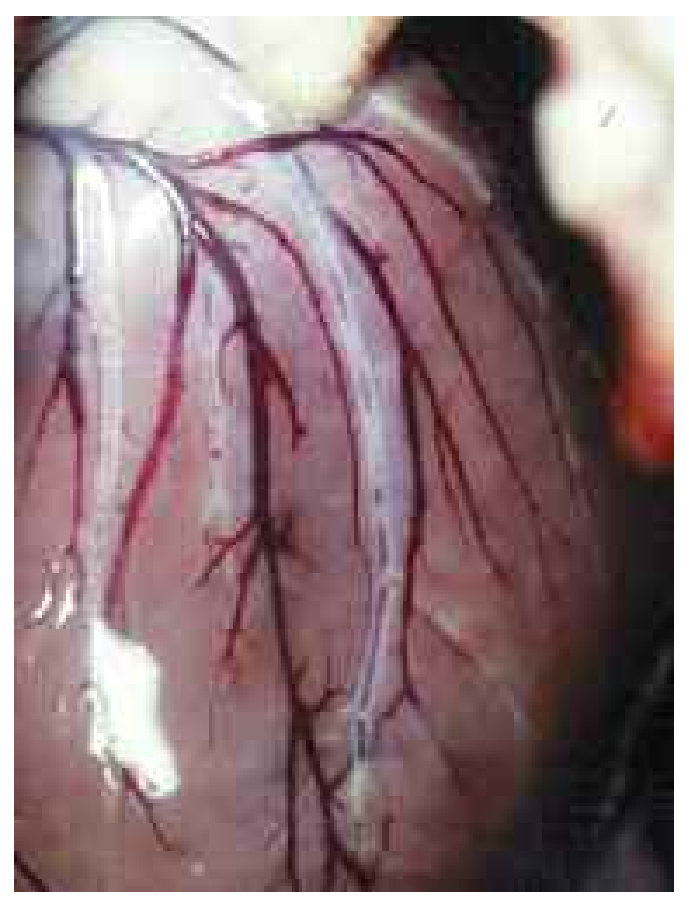

Fig 4. Air emboli in coronary blood vessels after blast exposure. 
with complete obstruction of the left anterior descending coronary artery has been reported following blast exposure (Keren 1981). In this case sub-intimal haemorrhage or intimal tearing by the blast wave may have predisposed to secondary thrombosis and subsequent infarction.

More commonly, myocardial injury is indirect and probably caused by coronary artery air emboli.

\section{Blood Vessels}

In blast lung, air can enter the systemic circulation via traumatic alveolo-venous fistulae. Any organ may be affected, but the cerebral and coronary circulations are of most relevance clinically (Figure 4). Arterial air embolism has been considered to be one of the principal causes of immediate and early death due to primary blast injury. Features of air emboli should be sought early in the assessment of the blast casualty and include:

- Cardiac arrhythmias and other evidence of myocardial ischaemia or infarction on electrocardiography. Abnormalities of cardiac rhythm may also arise indirectly through centrally mediated autonomic dysfunction from cerebral emboli;

- Headaches, vertigo, ataxia;

- Convulsions;

- Alterations in conscious level;

- Weakness or sensory loss;

- Facial or tongue blanching;

- Retinal artery air emboli.

The immediate action must be the administration of oxygen. It was previously thought that affected patients should be placed in the head-down position to reduce embolus flow into the coronary and cerebral circulations. Currently this advice has been abandoned, since there is evidence that the Trendelenberg position may promote gas embolism to the coronary vasculature and increase intracranial pressure. The definitive treatment of systemic air embolus is hyperbaric oxygen therapy, although this is not universally available. Hyperbaric oxygen therapy reduces the volume of gas bubbles and improves blood flow to hypoperfused tissues. Treatment should be instituted as soon as possible following blast exposure.

Disseminated intravascular coagulation has occurred in association with blast lung in a small series of five patients, of whom four additionally developed hypokalaemia (Melzer et al 1986). Since all five victims had other significant injuries, it is not possible to attribute these associations solely to blast lung.

Transplant teams considering organ harvest from brain dead patients who have sustained blast injuries need to be aware that air embolism may render apparently normal organs unusable. Massive compressed air emboli in the aorta and renal arteries have developed during ventilation for organ harvest. This has resulted in dissection of the interstitial tissues of the renal papillae (by air) and compression of collecting ducts (Freund et al 1980).

\section{Abdominal and Pelvic Organs}

Gas containing parts of the gastro-intestinal tract are at risk of primary blast injury, particularly in cases of immersion blast. Isolated primary blast injury to the gut is less frequent in air-blast. At high overpressures, immediate rupture of the gut wall occurs with bleeding and spillage of intraluminal contents into the peritoneal cavity. More commonly and at lower overpressures, haemorrhage develops within the intestinal wall (Figure 5).

These haemorrhages range in size from small petechiae to large confluent haematomas. Bleeding is initially confined to submucosal regions; with increasing severity it becomes transmural (Goligher et al 1943). These lesions demonstrate histological similarities to those seen in non-penetrative abdominal trauma and some will progress to necrosis of the gut wall and late perforation. The mesentery may also be involved; an injury undoubtedly caused by local shear motion of the bowel segment with respect to the restrained mesentery (Figure 6).

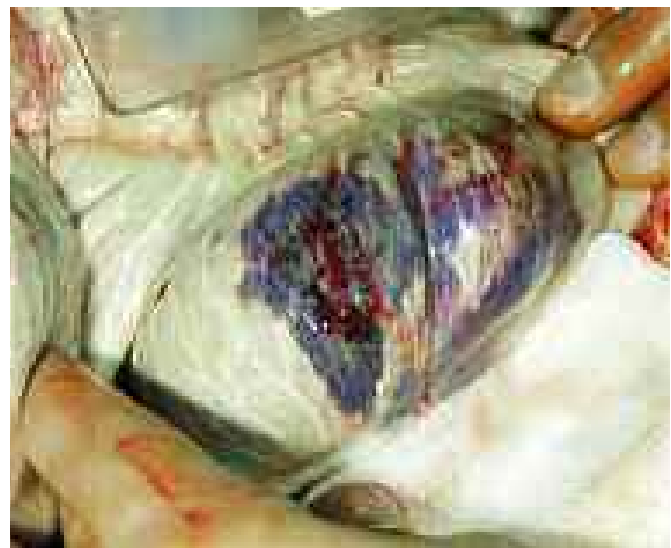

Fig 5. Contusion to large bowel caused by a blast wave; the injury was probably caused by intra-abdominal stress waves.

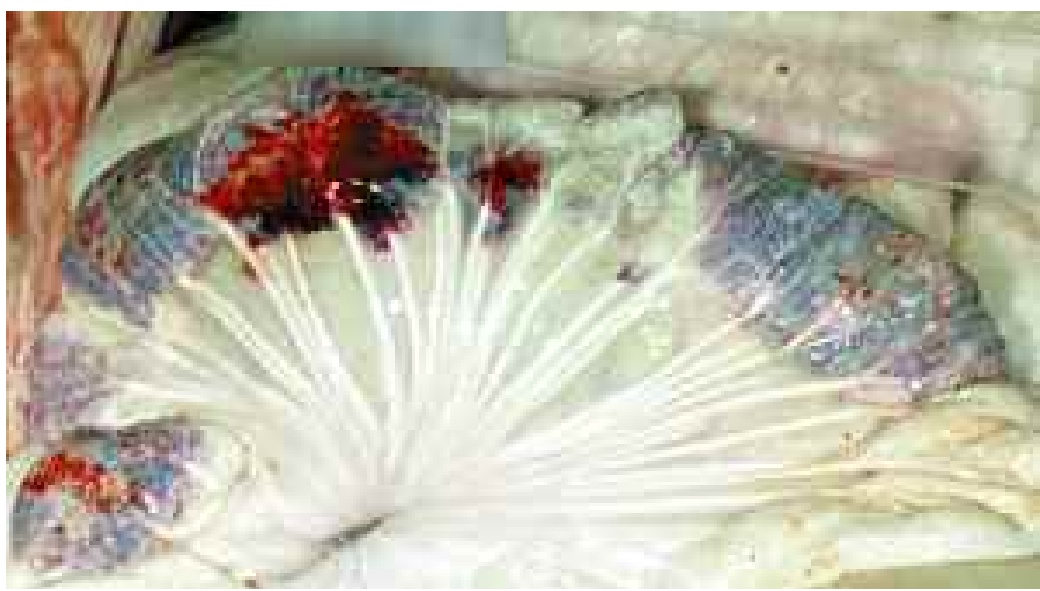

Fig 6. Damage to bowel and mesentery caused by a blast wave; the injury was probably caused by shear. 
Recent experimental studies on pigs (Cripps et al 1997) identified those contusions at greater risk of late perforation. At laparotomy, small bowel contusions $>15$ $\mathrm{mm}$ diameter and colonic contusions $>20$ $\mathrm{mm}$ diameter were at higher risk and it was considered that these warranted resection; smaller lesions could be treated conservatively.

Subcapsular haematomas, lacerations and fracture of solid organs (e.g. liver, spleen), testicular rupture and retroperitoneal haematomas have all been described in the literature. In the context of primary blast injury they result from high blast loads.

The clinical features of abdominal primary blast injury are listed in Table 6 . In some patients, the indications for exploratory laparotomy are obvious; in others primary blast injury to the abdomen represents a diagnostic challenge, since it may be clinically silent until complications are advanced. It is not the intention of this review to discuss in detail the surgical management of abdominal primary blast injury. However indications for urgent laparotomy would include:

- Unexplained signs of haemorrhage in patients who cannot be stabilised and in whom intra-abdominal injury is suspected;

- Radiological evidence of pneumoperitoneum (ruptured vicus);

- Rupture of the diaphragm;

- Obvious clinical evidence of peritoneal irritation;

- Significant and persistent bleeding from the gastro-intestinal tract.

Tension pneumoperitoneum is a rare complication of pulmonary barotrauma due to blast and causes dramatic changes in cardiovascular and respiratory function with severe hypoxaemia, hypercarbia and shock.

Table 7. Classification of Airborne Stimulation Hearing Los

1. Noise-Induced Hearing Loss (occupational hearing loss)

Long term exposure to increased sound intensities (generally $>85-90 \mathrm{~dB}$ ).

Bilateral and symmetrical sensorineural hearing loss which is initially reversible and most severe at $4 \mathrm{kHz}$.

Middle ear damage does not occur. Balance is not affected.

2. Report Trauma

Exposure to gunfire.

Stimuli are usually multiple.

Stimulus duration is less than $1.5 \mathrm{~ms}$.

Sensorineural hearing loss initially most marked

at $4 \mathrm{kHz}$; one ear is usually more severely affected.

Middle ear damage is possible, but unusual.

Vestibular features do not occur.

Caused by the positive phase of the blast wave. Stimulus duration is greater than $1.5 \mathrm{~ms}$.

Hearing loss may be sensorineural, conductive or mixed.

Middle ear damage is common. Vestibular symptoms may occur.
Table 6. Clinical Features of Abdominal Primary Blast Injury

Symptoms Abdominal pain.

Nausea, vomiting and (rarely) haematemesis.

Rectal pain, tenesmus.

Testicular pain.

Signs Abdominal tenderness, rebound and guarding.

Absent bowel sounds.

Evidence of hypovolaemia.

Intraperitoneal fibrosis is a late complication of abdominal primary blast injury. Small bowel adhesions, presenting as intestinal obstruction 50-60 years after blast exposure have been reported (Carter et al 1999).

\section{Orthopaedic Injuries}

Traumatic amputation of limbs by explosion is unusual in survivors $(1.5 \%)$, with the exception of those injured by anti-personnel mines, but relatively common in those who die early (19\%) (Mellor et al 1989).

It used to be considered that limb displacement (flailing) by the blast wind was the primary mechanism responsible for this injury. There is now substantial evidence that this is not the case. If traumatic amputation were due to avulsion of the limb by the dynamic overpressure alone, amputation should occur through or near joints. This occurs when ejecting fast jet pilots are exposed to slipstream wind speeds of 1100 $\mathrm{km} / \mathrm{h}$, which approximates to airspeeds caused by blast. An analysis of post-mortem data has revealed that traumatic amputations caused by explosions occur through long bone shafts and not in association with joints. Computer modelling studies and evidence from experiments on isolated goat hind limbs exposed to blast have supported a different mechanism of injury (Hull et al 1996). Coupling of the blast wave into bone tissue generates stress waves, which fracture the long bone shaft. Sites of predilection are the upper one third of the tibia and in the femur, either the upper or lower one third (depending on the type of explosive device). The dynamic overpressure generated by the blast wind separates the fractured limb from the body.

Large fragments (e.g. from vehicle bombs) may also cause traumatic amputation of limbs, but this is not the usual mechanism.

Death in individuals who have sustained traumatic amputation is usually attributable to severe associated secondary and tertiary injuries (e.g. open head injury, decapitation, large open chest wounds or evisceration), blast lung or exsanguination due to limb amputation.

\section{The Ear}

The auditory system is extremely sensitive to the blast wave. The middle ear cleft is gascontaining and the ear is uniquely constructed for the efficient transmission of 


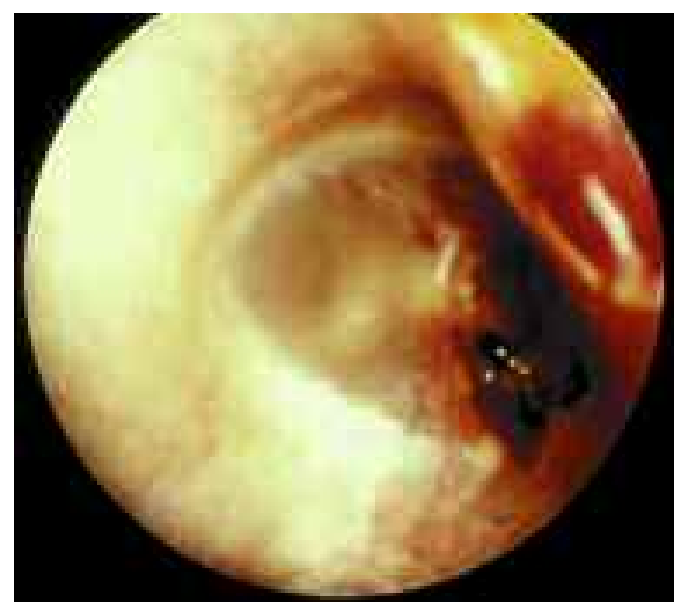

Fig 7. Rupture of the tympanic membrane.

mechanical energy (sound waves) and its transformation into nerve impulses. There has been confusion regarding the classification of airborne stimulation hearing loss. Noise-induced hearing loss, report trauma and blast trauma are separate clinical entities (Kerr 1987) (Table 7).

\section{External Ear}

The majority of injuries of the external ear are caused by penetrating and nonpenetrating fragments. Lacerations and similar injuries of the auricle are managed according to conventional principles.

\section{Middle Ear}

Tympanic membrane perforation is the most common significant middle ear injury (Figure 7). It is generally accepted that human tympanic membranes begin to rupture at pressures as low as $5 \mathrm{psi}(35 \mathrm{kPa})$ and that $50 \%$ will fail at $15 \mathrm{psi}(104 \mathrm{kPa})$ (Kerr 1978). Perforations invariably affect the pars tensa and vary from small defects in line with radial fibres of the lamina propria to complete loss of the tympanic membrane. These perforations are probably caused by the positive rather than the negative phase of the blast wave. This is supported by the following observations:

- Perforation edges are usually inverted;

- Medial displacement of ossicles;

- The phenomenon of implantation cholesteatoma following blast exposure;

- The presence of squamous epithelium in the middle ear cleft at post-mortem examination of specimens of temporal bones.

Perforation of the tympanic membrane, which is dependent on the orientation of the ear drum to the blast wave (amongst other factors), correlates poorly with serious primary blast injuries (to the lung or gut). In a study of 647 explosion survivors, of whom 193 had primary blast injury, $142(73.6 \%)$ had isolated tympanic membrane perforation and $18(9.3 \%)$ had blast lung in the absence of tympanic membrane rupture. Moreover, of those with isolated tympanic membrane rupture, none progressed to develop blast lung or intestinal primary blast injury isolated perforation of the tympanic membrane is not a marker for latent serious primary blast injury. Patients who present with this injury in the absence of clinical features of other blast injuries and who have a normal chest X-ray may be discharged after a short period of observation (Leibovici et al 1999).

At overpressures insufficient to perforate the eardrum, otoscopy may demonstrate hyperaemia (along the malleus handle or in the pars flaccida) or subepithelial bleeding. Associated ossicular injury is a feature of higher overpressures and has been reported as occurring in up to $33 \%$ of ears at tympanotomy (Sudderth 1974).

\section{Ossicular Injuries}

(Sudderth 1974; Wolf et al 1991; Chandler et al 1997)

These include:

- Incudo-malleolar joint disruption with medial displacement of the handle of the malleus (probably the most common ossicular injury);

- Incudo-stapedial joint disruption with or without fracture of the stapes superstructure;

- Fracture of the stapes superstructure alone;

- Fracture-dislocation of the stapes footplate;

- Dislocation of the incus;

- Erosion of the stapes superstructure or long process of the incus (thought to be due to avascular necrosis).

Although tympanic membrane perforation and disruption of the ossicular chain are considered to reduce cochlear damage by interfering with the transmission of mechanical energy from the tympanic membrane to the oval window, clinical evidence for this is lacking. Disruption of the stapes footplate or round window rupture with resultant leak of perilymph (perilymph fistula) occur rarely in humans (Nedzelski 1976). Isolated cases of secretory otitis media have been recorded following blast exposure. It is likely that these are incidental, at least in children in whom this condition is prevalent.

Cholesteatoma of the middle ear and mastoid air-cell system is a late complication occurring in up to $12 \%$ of blast induced perforations (Seaman et al 1971) and usually presenting up to 4 years after injury (Kronenberg et al 1988). It results from the implantation of small fragments of keratinizing squamous epithelium from the outer layer of the tympanic membrane into the middle ear cleft. If viable these cells develop into cholesteatoma pearls or invasive cholesteatomas histologically indistinguishable from primary or other acquired cholesteatomas. Since the majority of perforations caused by blast heal spontaneously, viable squamous epithelium 
with the potential to develop into cholesteatoma may be sequestered behind an intact tympanic membrane. It is for this reason that some authors advocate delay in surgical repair of tympanic membrane perforations which fail to heal spontaneously. The incidence of cholesteatoma formation is related to the grade of perforation (Kronenberg et al 1988) (Table 8).

Table 8.

\begin{tabular}{ll}
\hline Grade of Perforation & $\begin{array}{l}\text { Incidence } \\
\text { of } \\
\text { Cholestea- } \\
\text { toma (\%) }\end{array}$ \\
\hline 1 (up to 2 mm diameter) & 2.0 \\
2 (up to 25\% of the tympanic membrane) & 4.5 \\
3 (up to 50\% of the tympanic membrane) & 14.3 \\
4 (subtotal perforation) & 20.0 \\
\hline
\end{tabular}

Of particular interest is the observation that marginal perforations caused by blast are not associated with a higher incidence of cholesteatoma. Whether invasive cholesteatoma caused by blast is best treated by combined approach tympanoplasty (CAT, canal wall up procedure) or by open cavity mastoidectomy (canal wall down approach), is open to debate. Multifocal implantation cholesteatoma of the external auditory canal is a much rarer complication (Wolf et al 1999).

\section{Inner Ear}

Blast exposure often causes sensorineural hearing loss with high pitched tinnitus. In many cases these resolve spontaneously, sometimes within hours. The prevalence of permanent sensorineural hearing loss varies between 30 and 55\% (Chandler et al 1997). There is wide variation in the audiometric configuration, but the most common pattern on pure tone audiometry is that of a high frequency loss. A notch at $4 \mathrm{kHz}$ which is characteristic of noise induced hearing loss and report trauma does not occur. Injury to the cochlea by the blast wave is considered to be a mechanical injury to sensory structures on the basilar membrane with tearing of sensory cells from their supporting cell attachments due to excessive displacement of the basilar membrane (Patterson et al 1997).

Reversible discontinuities in the reticular lamina are thought to be responsible for temporary threshold shifts. These may include disruption of tight junctions between Hensen cells, changes in membrane permeability and holes in the reticular lamina. These result in mixing of perilymph and endolymph. When these breaches are repaired and the electrochemical properties of inner ear fluid systems restored, normal function may be re-established. Loss of hair cells, structural abnormalities of cilia and detachment of long segments of the organ of Corti probably account for permanent threshold shifts.

Measurements of auditory brainstem evoked potentials following blast exposure indicate that central auditory pathways function normally at least in survivors (Pratt 1985).

Vertigo is unusual following blast. A small number of cases of benign paroxysmal positional vertigo have been recorded, suggesting damage to the utricle but most cases of vertigo or dysequilibrium have been attributed to associated head injury.

\section{Clinical Features}

It is easy to miss blast injury of the ear in a severely injured patient. Otological symptoms and signs (Table 9) should be sought on assessment of blast injured individuals.

Table 9. Clinical Features of Primary Blast Injury of the Ear

- Hearing loss and tinnitus (occur in most patients, at least initially and usually improve, but may be permanent).

- Otalgia (temporary, but may last for several weeks).

- Vertigo is uncommon.

- Bleeding from the ear canal due to perforation of the tympanic membrane.

- Tympanic membrane perforation (typically slit-like or punched-out in shape with irregular edges and tiny blood clots attached to the margins).

- Mucopurulent otorrhoea is a sign of secondary infection of the middle ear cleft.

Fracture of the petrous temporal bone (due to secondary or tertiary blast injury) should be excluded in patients with bleeding from the ear canal. Severe vertigo with horizontal nystagmus, CSF otorrhoea, facial nerve paralysis or Battle's sign suggest temporal bone fracture. A normal plain skull radiograph does not exclude a fracture of the skull base; cases of complicated skull base fracture will require sophisticated imaging which may include computed tomography, magnetic resonance imaging or angiography.

\section{Management}

All patients exposed to blast should have an otological assessment and audiometry.

Over $80 \%$ of tympanic membrane perforations caused by blast are likely to heal spontaneously (Kerr et al 1975; Pahor 1981). Large perforations ( $>80 \%$ surface area) are less likely to heal without surgery. Although some authors advocate immediate repair, (Ziv et al 1973), most otologists treat these perforations expectantly, waiting up to 12 months before undertaking elective tympanoplasty. During this period ears should be kept dry.

Antibiotics (topical or systemic) are not indicated except in the presence of infection. Early surgical intervention is confined to the removal of foreign material and debris from the ear canal, although a good case can be made for the approximation of perforation edges (Gapany-Gapanavicius et al 1977). The outcome of surgery to repair tympanic membrane perforations caused by blast, is excellent; $90 \%$ of grafted tympanic 
membranes will heal completely.

There is little evidence that vasodilators, low molecular weight dextrans, anti-platelet drugs, vitamins or systemic steroids improve the outcome for patients with sensorineural hearing loss caused by blast. It remains current practice in this country to administer one or a combination of these drugs to patients with sudden sensorineural hearing loss on an inpatient basis.

Patients with blast induced perforations are at risk of late presenting implantation cholesteatoma and therefore require long term review.

The diagnosis of a perilymph fistula should be considered in a patient with sensorineural hearing loss and vertigo, particularly if either of these is fluctuating, since the treatment is surgical (urgent exploratory tympanotomy and repair of the perilymph leak).

\section{The Eye and Orbit}

Although the eyeball represents only $0.1 \%$ of the body surface area, ocular injuries caused by explosions are relatively frequent (with up to $10 \%$ of blast survivors being affected). The majority are produced by small projectiles to which the eye is extremely vulnerable. Blast fragmentation from munitions accounted for $68-86 \%$ of all ophthalmic casualties in various conflicts from WWI to Operation Desert Storm.

The globe is relatively resistant to the direct action of the blast wave, since its constituent parts are of similar density. Therefore the incidence of primary blast injury to the eye is likely to be low, at least in survivors. A single case of conjunctival injection and hyphaema caused by blast in the definite absence of either penetrating or blunt trauma has been reported (Beiran et al 1992).

More recent experimental studies (Petras et al 1997) have shown evidence of retinal injury and degeneration of central visual pathways (retinofugal axonopathy) in rats exposed to blast.

Blow-out fractures of the orbit can be produced in experimental animals (dogs) at lethal levels of blast overpressure, but these are rare in humans. There is one clinical case cited in the literature in which bilateral blow-out fractures involving the orbital floor with extrusion of the left eyeball into the maxillary sinus and severance of the optic nerve resulted from blast (Mandelcorn et al 1973).

Air emboli caused by pulmonary primary blast injury, and present in retinal arteries probably explain transient episodes of blindness.

\section{Central Nervous System}

Head injury due to the secondary or tertiary effects of blast, frequently contributes to death in both immediate $(71 \%)$ and late (52\%) fatalities (Frykberg et al 1988). There is increasing evidence that the central nervous system is at risk of blast injury by other mechanisms. The occurrence of air emboli in the cerebral circulation following blast is well known. Early anecdotal reports and recent experimental studies implicate the primary effect of blast as a mechanism of injury to the brain (Kaur et al 1995).

\section{Removal of Unexploded Ordnance}

Patients with retained unexploded ordnance are rare, but present a considerable logistical and surgical challenge. Reports of these injuries to date have been confined to the military literature. Weapons most commonly implicated are the M79 grenade-launcher (40 $\mathrm{mm}$ round) and mortars $(48-82 \mathrm{~mm}$ rounds). In a recent review of 32 such patients injured in conflicts from WW II to Somalia (1993) and who survived surgical removal of unexploded ordnance, a strategy for the management was proposed (Lein et al 1999)(Table 10).

Table 10. Management of Patients with Unexploded Ordnace

- Notification of the incident to the Explosive Ordnance Disposal (EOD) team.

- The use of closed cardiac massage and defibrillation, which may detonate the round, should be avoided in patients who are moribund.

- Isolation of the patient from the rest of the hospital to a protected operating area for removal of the ordnance to minimise collateral damage in the event of premature detonation. Sandbag barriers and body armour should be employed.

- Strong electrical fields, vibration, temperature fluctuations and changes in position or orientation of the munition may arm the fuse. The use of electrocautery, powered instrumentation and other similar electrical equipment should be avoided and patient movement minimised.

- Plain radiography should be used in preference to more sophisticated imaging modalities (e.g. computed tomography, magnetic resonance imaging, ultrasound), which are more likely to trigger the fuse. - En bloc removal of the munition with surrounding tissues expedites surgery and reduces the probability of contact between the round and metal instruments which may cause detonation. Following removal the patient can be transferred to a more appropriate operating room for definitive surgery and further management.

\section{Conclusions}

The relevance of blast injuries to military doctors is inescapable, but explosions are not confined to the battlefield. Industrial accidents and detonation of terrorist devices are occasional events capable of generating large numbers of casualties whose injuries may be unfamiliar to many physicians. An understanding of the mechanisms of these injuries and their management should be of assistance in the aftermath of such an event. Interest in explosions and their effects has formerly been confined to the military; today all doctors should be cognisant of the injuries caused by blast. Key points in the management of blast casualties are presented in Table 11. 
Table 11. Key Management Points

- Primary blast injury should be considered in all individuals exposed to blast.

- Serious primary blast injuries are uncommon in survivors; fragmentation injuries predominate.

- Blast lung presents early. Diagnosis is made on clinical grounds and confirmed on plain chest radiography. This diagnosis needs to be excluded in patients who require general anaesthesia or transportation in partially pressurised aircraft. Prophylactic chest drain insertion is advocated in patients with blast lung under these circumstances.

- Isolated perforation of the tympanic membrane is not a marker for life threatening primary blast injuries.

- Abdominal primary blast injury may be clinically silent until complications (including peritonitis and septicaemia) are advanced.

- Patients who sustain traumatic amputation of limbs, should they survive to reach hospital, often have multiple life threatening injuries.

- Primary blast injuries of the auditory system are easily overlooked and are a source of significant morbidity and future litigation. They should be accurately documented and promptly referred for specialist management.

\section{References}

Beiran I, Miller B. Pure ocular blast injury. $A m \mathcal{f}$ Ophthalmol. 1992; 114: $504-505$.

Benzinger T. Physiological effects of blast in air and water. In German Aviation Medicine World War II, Volume II, Chapter XIV-B, pages 1225 - 1259. U.S. Department of the Air Force.

Brown RFR, Cooper GJ, Maynard RL. The ultrastructure of rat lung following acute pulmonary blast injury. Int $\mathcal{F}$ Exp Path. 1993; 74: 151 - 162.

Carter PS, Belcher PE, Leicester RJ. Small bowel adhesions long after blast injury. $f R$ Soc Med. 1999; 92: 135 - 136 .

Caseby NG, Porter MF. Blast injuries to the lungs: clinical presentation, management and course. Injury 1976; 8: 1 - 12 .

Chandler DW, Edmond CV. Effects of blast overpressure on the ear: case reports. $7 \mathrm{Am}$ Acad Audiol. 1997; 8: 81 - 88 .

Chiffelle TL. Pathology of direct air-blast injury. Technical progress report on contract no. DA - 49 146 - XZ - 055. 1966. Lovelace Foundation for Medical Education and Research, Albuquerque, New Mexico.

Cohn SM. Pulmonary contusion: a review of the clinical entity. F Trauma 1997; 42: 973 - 979.

Cooper GJ, Taylor DEM. Biophysics of impact injury to the chest and abdomen. F R Army Med Corps 1989; 135: $58-67$.

Coppel DL. Blast injuries of the lungs. Br F Surg. 1976; 63: $735-737$.

Cripps NPJ, Cooper GJ. Risk of late perforation in intestinal contusions caused by explosive blast. $\mathrm{Br} F$ Surg. 1997; 84: 1298 - 1303.

Freund U, Kopolovic J, Durst AL. Compressed air emboli of the aorta and renal artery in blast injury. Injury 1980; 12: 37 - 38 .

Frykberg ER, Tepas JJ. Terrorist bombings. Lessons learned from Belfast to Beirut. Ann Surg. 1988; 208: $569-576$.

Gapany-Gapanavicius B, Brama I, Chisin R. Early repair of blast ruptures of the tympanic membrane. $\mathcal{F}$ Laryngol Otol. 1977; 91: 565 - 573.

Goligher JC, King DP, Simmons HT. Injuries produced by blast in water. Lancet 1943 (2): 119 123.

Guy RJ, Kirkman E, Watkins PE, Cooper GJ. Physiologic responses to primary blast. F Trauma 1998; 45: 983 - 987.

Hill JF. Blast injury with particular reference to recent terrorist bombing incidents. Ann R Coll Surg Engl. 1979; 61: 4-11.

Hirshberg B et al Recovery from blast lung injury: oneyear follow-up. Chest 1999; 116: 1683 - 1688.
Hull JB, Cooper GJ. Pattern and mechanism of traumatic amputation by explosive blast. I Trauma 1996; 40: S198 - 205.

Iremonger MJ. Physics of detonations and blast waves. In: Cooper GJ, Dudley HAF, Gann DS, Little RA, Maynard RL eds. Scientific foundations of trauma. Butterworth-Heinemann. 1997: 189 - 199.

Katz E, Ofek B, Adler J, Abramovitz, HB, Krausz MM. Primary blast injury after a bomb explosion in a civilian bus. Ann Surg. 1989; 209: 484 - 488.

Kaur C, Singh MK, Lim B, Ng BL, Yap EPH, Ling EA. The response of neurons and microglia to blast injury in the rat brain. Neuropathology and Applied Neurobiology 1995; 21: $369-377$.

Keren A, Stessman J, Tzivoni D. Acute myocardial infarction caused by blast injury of the chest. Br Heart f. 1981; 46: 455 - 457.

Kerr AG. Blast injuries to the ear. Practitioner 1978; 221: $677-682$.

Kerr AG. Blast injury to the ear: a review. Rev Environ Health 1987; 7: 65 - 79 .

Kerr AG, Byrne JET. Concussive effects of bomb blast on the ear. F Laryngol Otol. 1975; 89: 131 - 143.

Kronenberg J, Ben-Shoshan J, Modan M, Leventon G. Blast injury and cholesteatoma. Am f Otol.1988; 9: 127 $-130$.

Laure P. Methaemoglobinaemia: an unusual case report. Intensive Care Med. 1993; 19: 124

Leibovici D, Gofrit ON, Shapira SC. Eardrum perforation in explosion survivors: is it a marker of pulmonary blast injury? Ann Emerg Med. 1999; 34; 168 $-172$.

Lein B, Holcomb J, Brill S, Hetz S, McCrory T. Removal of unexploded ordnance from patients: A 50year military experience and current recommendations. Mil Med. 1999; 164: 163 - 165.

Mandelcorn MS, Hill JC. Orbital blast injury. Canad $\mathcal{F}$ Ophthal. 1973; 8: 597 - 599.

Mellor SG, Cooper GJ. Analysis of 828 servicemen killed or injured by explosion in Northern Ireland 1970 - 84: the Hostile Action Casualty System. Br F Surg. 1989; 76: 1006 - 1010 .

Melzer E, Hersch M, Fischer D, Hershko C. Disseminated intravascular coagulation and hypopotassemia associated with blast lung injury. Chest 1986; 89: $690-693$.

Nedzelski JM, Barber HO. Round window fistula. $\mathcal{f}$ Otolaryngol. 1976; 5: $379-381$.

Oppenheim A, Pizov R, Pikarsky A et al. Tension pneumoperitoneum after blast injury: dramatic improvement in ventilatory and hemodynamic parameters after surgical decompression. $f$ Trauma 1998; 44: 915 - 917

Pahor AL. The ENT problems following the Birmingham bombings. F Laryngol Otol. 1981; 95: 399 $-406$.

Patterson JH, Hamernik RP. Blast overpressure induced structural and functional changes in the auditory system. Toxicology 1997; 121: $29-40$.

Petras JM, Bauman RA, Elsayed NM. Visual system degeneration induced by blast overpressure. Toxicology 1997; 121: 41 - 49 .

Pizov R, Oppenheim-Eden A, Matot I et al. Blast lung injury from an explosion on a civilian bus. Chest 1999; 115: $165-172$.

Pratt H, Goldsher M, Netzera A, Shenhav R. Auditory brainstem evoked potentials in blast injury. Audiology 1985; 24: 297-304.

Schild HH, Strunk H, Weber W et al. Pulmonary contusion: CT vs plain radiograms. $\mathcal{F}$ Comput Assisted Tomogr. 1989; 13: 417 - 420.

Seaman RW, Newell RC. Another etiology of middle ear cholesteatoma. Arch Otolaryngol. 1971; 94: 440 442.

Sorkine P, Szold O, Kluger Y et al. Permissive hypercapnia ventilation in patients with severe pulmonary blast trauma. F Trauma 1998; 45: $35-38$.

Sudderth ME. Tympanoplasty in blast-induced perforation. Arch Otolaryngol. 1974; 99: 157 - 159. Uretzky G, Cotev S. The use of continuous positive airway pressure in blast injury of the chest. Crit Care Med. 1980; 8: 486 - 489 . 
Wagner RB, Jamieson PM. Pulmonary contusion: Evaluation and classification by computed tomography. Surg Clin North Am. 1989; 69: 31 - 40. Wang CY, Yap BH, Delilkan AE. Melioidosis pneumonia and blast injury. Chest 1993; 103: 1897 1899.

Wolf M, Ben-Shoshan J, Kronenberg J, Roth Y. Blast injury of the ear. Mil Med. 1991; 156: $651-653$.
Wolf M, Megirov L, Kronenberg J. Multifocal cholesteatoma of the external auditory canal following blast injury. Ann Otol Rhinol Laryngol. 1999; 108: 269 -270 .

Ziv M, Leventon G, Philipsohn NC, Man A. Blast injury of the ear: Treatment and evaluation. Mil Med. 1973; 138: $811-813$.

Zuckerman S. Experimental study of blast injuries to the lungs. Lancet 1940; (2): $219-224$. 